\title{
MASS MOVEMENTS’ DETECTION IN HIRISE IMAGES OF THE NORTH POLE OF MARS
}

\author{
L. Fanara ${ }^{\text {a, b, * }}$, K. Gwinner ${ }^{\text {a }}$, E. Hauber ${ }^{\text {a }}$, J. Oberst ${ }^{\text {a, b }}$ \\ ${ }^{\text {a }}$ Institute of Planetary Research, DLR, Berlin, Germany - (Lida.Fanara, Klaus.Gwinner, Ernst.Hauber, Juergen.Oberst)@dlr.de \\ ${ }^{\mathrm{b}}$ Institute of Geodesy and Geoinformation Science, Technical University of Berlin, Germany
}

Commission IV, WG IV/8

KEY WORDS: Change detection, Mass Movements, Mars, HiRISE

\begin{abstract}
:
We are investigating change detection techniques to automatically detect mass movements at the steep north polar scarps of Mars, in order to improve our understanding of these dynamic processes. Here we focus on movements of blocks specifically. The precise detection of such small changes requires an accurate co-registration of the images, which is achieved by ortho-rectifying them using High Resolution Imaging Science Experiment (HiRISE) Digital Terrain Models (DTMs). Moreover, we deal with the challenge of deriving the true shape of the moved blocks. In a next step, these results are combined with findings based on HiRISE DTMs from different points in time in order to estimate the volume of mass movements.
\end{abstract}

\section{INTRODUCTION}

Hundreds of individual mass movement events have been observed since 2006, when High Resolution Imaging Science Experiment (HiRISE) started imaging in Mars orbit (McEwen et al., 2007). Since then, some regions have been imaged with a frequency of up to 7 times per month. This does not only allow us to detect the changes but also to narrow down the time interval within which they occurred. In this study we focus on movements of blocks, which represent the most prominent result of rock fall processes occurring at the steep north polar scarps and are also the most common evidence for mass movements in this area.

Blocks have been found to appear as well as disappear in specific places. In some cases they seem to have disintegrated into smaller pieces while moving downslope. Due to their small sizes blocks are only visible in HiRISE images. They originate at the steep margins of the north polar cap, where their point of origin is sometimes clearly visible either at the North Polar Layered Deposits (NPLD) or at the Basal Unit (BU) (Russel et al., 2014).

In order to track and quantify these movements we are investigating suitable techniques for identifying new and disintegrated blocks in images taken at different times.

\section{CHANGE DETECTION}

Wagstaff et al. (2012) and Di et al. (2013) dealt with largerscale changes, such as impact craters and dark slope streaks. Sidiropoulos and Muller (2015) attempt a more generalized approach for all kinds of change based on multiple types of images. However, mass movements such as block falls involve small objects, a couple of cubic meters in volume, which are only visible in HiRISE images, and therefore require a processspecific approach with high geometric accuracy.

Figure 1 shows our work process. We started by analysing the HiRISE image coverage of the steep margins of the north polar cap both temporally and in terms of stereo possibility. Due to the high rate of imaging at the north pole, there are multiple images which comprise stereo pairs and can be used to detect differences also in 3D.

We produce HiRISE Digital Terrain Models (DTMs) using the Ames Stereo Pipeline (ASP) (Moratto et al., 2010) in order to accurately locate the events by ortho-rectifying the images on these DTMs. The resulting DTMs and co-registered images form the basis for 3D and 2D change detection. DTM-based mapping of height changes, however, is an extremely challenging task due to the requirements on the resolution and accuracy of co-registration in an area characterised by steep slopes.

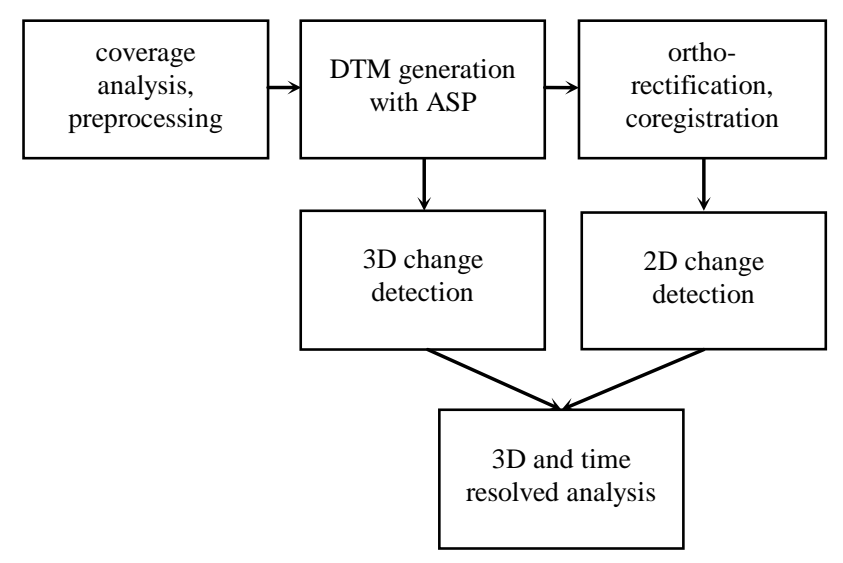

Figure 1. Work process 
We are currently developing a method for detecting displaced blocks in areas like the steep north polar scarp in Figure 2. A large number of different types of blocks appeared within 4 years at the foot of the scarp (Figure 3). The source of these blocks could be the changes at the scarp (Figure 2, black arrows).

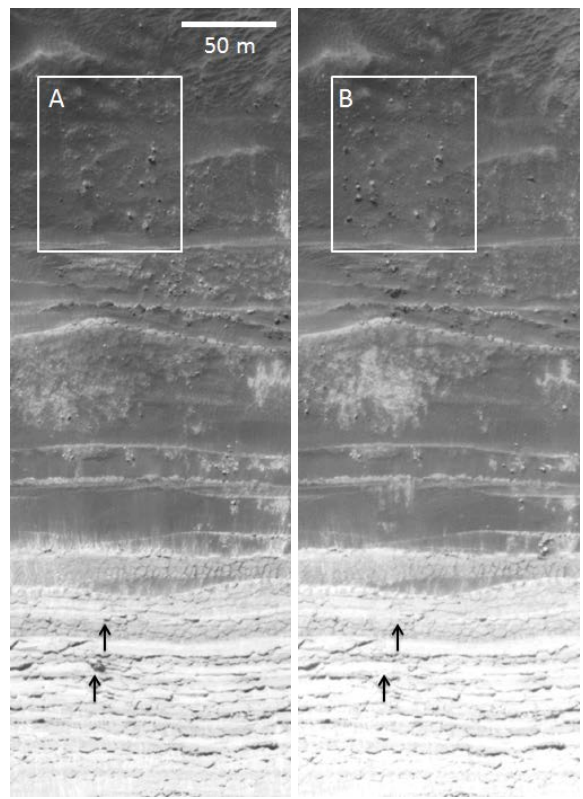

Figure 2. North polar steep scarp with 4 years difference

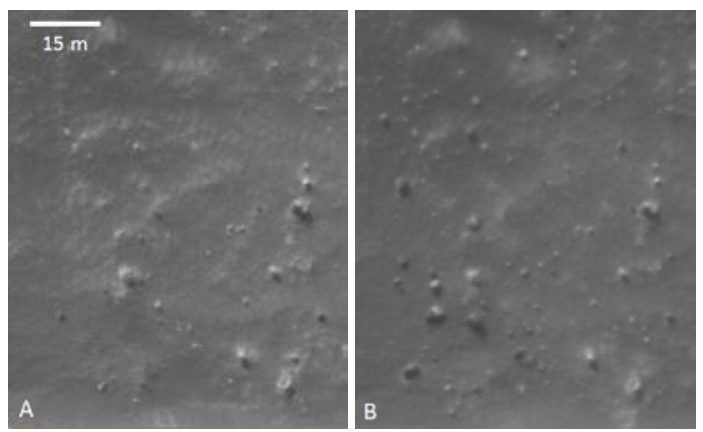

Figure 3. Test area with 4 years difference

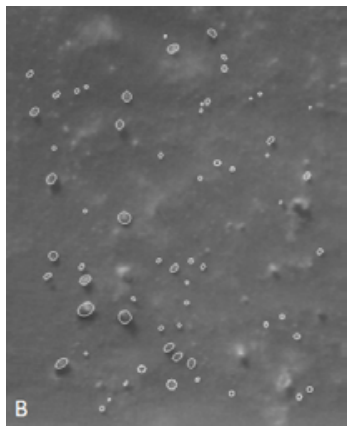

Figure 4. Desired change detection result

Figure 4 represents the desired change detection result which we are aiming to achieve. To do this we are testing a simple structure-based method, where we apply an edge detection filter on both images and subtract them. In order to isolate only the meaningful changes we apply thresholding to the difference image and morphological opening to obtain closed surface areas corresponding to blocks. This simple method succeeds in finding the new blocks between the images, but fails in recognising accurately their shape and size.

\section{CONCLUSIONS}

We have set up a systematic procedure for producing accurately co-registered HiRISE images. Simple change detection methods succeed in detecting the moved blocks. However, the size and shape of the blocks are not recognised with sufficient accuracy. Nevertheless, this method may be used to indicate the approximate location of new blocks with a different, more sophisticated method to detect them as ellipsoidal objects comprised of a light region with a shadow.

We believe our final results will help to improve our understanding of the processes and volumes involved in the mass movements and thus the evolution of the north polar cap.

\section{ACKNOWLEDGEMENTS}

This work is co-funded by the European Union's Seventh Framework Programme under iMars grant agreement $n^{\circ}$ 607379 .

\section{REFERENCES}

Di, K., Liu, Y., Hu, W., Yue, Z., and Liu, Z., 2013. Mars surface change detection from multi-temporal orbital images. In: $35^{\text {th }}$ International Symposium on Remote Sensing of Environment, Beijin, China.

McEwen, A. S., Eliason, E. M., Bergstrom, J. W., Bridges, N. T., Hansen, C. J., Delamere, W. A., Grant, J. A., Gulick, V. C., Herkenhoff, K. E., Keszthelyi, L., Kirk, R. L., Mellon, M. T., Squyres, S. W., Thomas, N., and Weitz, C. M., 2007. Mars Reconnaisance Orbiter's High Resolution Imaging Science Experiment (HiRISE). Journal of Geophysical Research, 112 (E5)

Moratto, Z. M., Broxton M. J., Beyer R. A., Lundy M., and Husmann K., 2010. Ames Stereo Pipeline, NASA's Open Source Automated Stereogrammetry Software. In: $41^{\text {st }}$ Lunar and Planetary Science Conference, The Woodlands, Texas, USA.

Russell, P. S., Feleke, S., and Byrne, S., 2014. Landslide erosion rates of north polar layered deposit cliffs and the underlying basal unit. In: Eighth International Conference on Mars, Pasadena, California, USA.

Sidiropoulos, P. and Muller J.-P., 2015. Identifying dynamic features on Mars through multi-instrument co-registration of orbital images. In: European Planetary Science Congress, Nantes, France.

Wagstaff, K. L., Panetta, J., Ansar, A., Greeley, R., Pendleton Hoffer, M., Bunte, M., and Schörghofer, N., 2012. Dynamic landmarking for surface feature identification and change detection. In: ACM Transactions on Intelligent Systems and Technology, Vol. 3, pp. 49:1-49:22. 